\title{
A rule dynamics approach to event detection in Twitter with its application to sports and politics
}

Article

Accepted Version

Creative Commons: Attribution-Noncommercial-No Derivative Works 4.0

Adedoyin-Olowe, M., Gaber, M. M., Dancausa, C., Stahl, F. and Gomes, J. B. (2016) A rule dynamics approach to event detection in Twitter with its application to sports and politics. Expert Systems with Applications, 55. pp. 351-360. ISSN 0957-4174 doi: https://doi.org/10.1016/j.eswa.2016.02.028 Available at https://centaur.reading.ac.uk/78776/

It is advisable to refer to the publisher's version if you intend to cite from the work. See Guidance on citing.

Published version at: http://www.open-access.bcu.ac.uk/3827/

To link to this article DOI: http://dx.doi.org/10.1016/j.eswa.2016.02.028

Publisher: Elsevier

All outputs in CentAUR are protected by Intellectual Property Rights law, including copyright law. Copyright and IPR is retained by the creators or other copyright holders. Terms and conditions for use of this material are defined in the End User Agreement.

www.reading.ac.uk/centaur 
Central Archive at the University of Reading

Reading's research outputs online 


\title{
A Rule Dynamics Approach to Event Detection in Twitter with Its Application to Sports and Politics
}

\author{
Mariam Adedoyin-Olowe, Mohamed Medhat Gaber, Carlos M. Dancausa \\ School of Computing Science and Digital Media, Robert Gordon Univeristy \\ Aberdeen, AB10 7GJ, UK \\ m.a.adedoyin-olowe@rgu.ac.uk,m.gaber1@rgu.ac.uk, c.j.martin-dancausa@rgu.ac.uk \\ Frederic Stahl \\ School of Systems Engineering, University of Reading \\ PO Box 225, Whiteknights, Reading, RG6 6AY, UK \\ F.T.Stahl@reading.ac.uk \\ João Bártolo Gomes \\ DataRobot, Inc. \\ Singapore \\ joao@datarobot.com
}

\begin{abstract}
The increasing popularity of Twitter as social network tool for opinion expression as well as information retrieval has resulted in the need to derive computational means to detect and track relevant topics/events in the network. The application of topic detection and tracking methods to tweets enable users to extract newsworthy content from the vast and somehow chaotic Twitter stream. In this paper, we apply our technique named Transaction-based Rule Change Mining to extract newsworthy hashtag keywords present in tweets from two different domains namely; sports (The English FA Cup 2012) and politics (US Presidential Elections 2012 and Super Tuesday 2012). Noting the peculiar nature of event dynamics in these two domains, we apply different time-windows and update rates to each of the datasets in order to study their impact on performance. The performance effectiveness results reveal that our approach is able to accurately detect and track newsworthy content. In addition, the results show that the adaptation of the time-window exhibits better performance especially on the sports dataset, which can be attributed to the usually shorter duration
\end{abstract}


of football events.

Keywords: Twitter, Hashtags, Associations Rules, Rule Matching,

Rule Mapping

\section{Introduction}

There has being a surge in Twitter activities [1] since its launch in 2006 as well as steady increase in event detection awareness on the network [2] in recent times. With over 645 million subscribers [3], Twitter has continued to grow both

5 in size and activity. The network is rapidly changing the way the global audience sources for information and thus influences the process of journalism [4, 5]. Twitter is becoming an information network rather than just a social media when compared with other social networks such as Facebook and Tumblr. This explains why traditional news media follow activities on Twitter to enhance their news reports and updates. News media like $B B C$ or $C N N$ that contain full story they broadcast on their Twitter page thereby giving readers the opportunity of reading the full story. Breaking news are sometimes posted on Twitter before they are published by traditional news media due to users' nearness to the location of events 6, 7. An example of such a situation is the news of the death

15 of America's female pop star Whitney Houston, which was posted on Twitter before its broadcast on news media 8 as a breaking news. The dynamic and streaming nature of Twitter data (known as tweet) also includes noise on the network resulting in the difficulty of manually obtaining meaningful contents from Twitter. Where some tweets are relevant to specific real life events and are worthy of being broadcasted, others constitute noise [9]. It shows the need for filtering in order to extract relevant tweets from Twitter. According to Allan [10, a topic as defined in Topic Detection and Tracking (TDT) context can be " a set of news stories that are strongly related by some similar events". Events often trigger topics; for instance, breaking news about the sudden death of a public figure will trigger other related news such as police investigations into the cause of death, trail of suspects, arrest and trials of suspect. All these unfolding 
events will generate news updates and result in the evolvement of related topics. TDT methods are currently used to detect and track trending events on Twitter over time [11.

so In our previous work [12, 13, we analysed hashtag keywords in tweets on the same topic at 2 consecutive time periods using Association Rule Mining (ARM) and Transaction-based Rule Change Mining (TRCM). Our TRCM method was able to identify 4 temporal Association Rules (ARs) relating to evolving concept of tweets. The identified ARs are namely; "New rules", "Emerging rules", 35 "Unexpected Consequent/Conditional rules" and "Dead rules". The results of our previous experiments [12, 13] relates the identified $A R s$ to evolving events in real life. To maintain coherence in this paper, ARM, ARs and TRCM concepts will be explained in subsequent sections.

In this paper we automate the detection of real life topics generated in 3 Twitter 40 datasets from 2 different domains; sports (the English FA Cup Final 2012) and politics (US Presidential Elections 2012 and US Super Tuesday 2012). We map all hashtag keywords extracted by our system during training process to related topics from carefully chosen ground truth to ascertain a match and subsequently to validate our system's performance. A match is said to have occurred if the time-slot of an extracted hashtag keyword correlates with the time of event occurrence in the ground truth. We evaluate how the dynamics of each dataset affects our experimental results. For performance effectiveness analysis of our method, we consider precision over recall. This is because we are more concerned with generating relevant hashtag keywords (precision) that are related to targeted real life topics/events. As far as we are aware of, TRCM is the only method that detects topics from Twitter using hashtags and ARM. We list the contributions of this paper as follows.

- Automation of event detection and tracking in Twitter in one cohesive computational framework, different compared with earlier work that separated event detection and tracking;

- Application of the proposed methods on datasets of different nature of 
dynamism (from the very dynamic in sports to the slow unfolding events in politics);

- Providing proof of universality of our proposed methods in a number of

\section{Related Work}

TDT methods can be used to extract interesting topics from Twitter streaming data and present patterns that demonstrate a representation of specific real life topics. This is achieved by mapping detected results to real life news/events high volume of data very rapidly, it is important to apply TDT to Twitter data in order to organise this large volume of data in a meaningful way. There is very limited work on the application of $A R M$ as TDT method on Twitter data. Diverse TDT methods are being used to detect relevant events and news topics embedded in online tweets. Events tweets are robust ranging from sports [14, 15], politics [16], stock market [17]. The N-grams method effectively captures intricate combination of tweets' keywords in real life topics of diverse composite and time scale by recognising the trend in the topics [11]. Other TDT methods are applied to tweets to analyse real life events and occurrences such as 
sparsely reported events [18, differentiating between real world events and nonevent tweets [19]. These methods are also capable of monitoring topic trends (emerging topics) on Twitter in real time [20]. Our method not only monitors the trend of emerging topics in real life, it also detects and tracks any changes in the flow of the detected topic or event. Scalable distributed event detection 17] as well as characterising emerging trends [21] have also been conducted on Twitter data. Similarly, TDT methods detect and track breaking news [22] and first mention of story often referred to as first story [23] on Twitter. Our method is holistic in that it detects and tracks different types of topics/events either breaking news or emerging stories. TDT methods are also trained to predict the outcome of national elections 24] and to detect local events posted on Twitter [25].

Becker et al [26] used an online clustering and filtering framework to distinguish between messages about real life events and non-events. The framework clusters subsequent tweet-based messages using their similarity with existing clusters. On the other hand, graph-based approaches can detect keyword clusters in tweets based on their pairwise comparison [27, 11. This can be a term unison graph with nodes clustered and the use of community detection algorithm based on betweenness centrality [28]. Graph-based methods can also be applied to evaluate the effectiveness of topic extraction from tweets [29]. Jackoway et al 30] used a clustering technique to detect events using a text classifier. Phuvipadawat and Murtata 22] proposed a method for collecting, grouping, ranking and tracking breaking news in Twitter. They built a framework named 'Hotstream' to enable users to discover breaking news from Twitter timeline. Other approaches considered first story detection on the network. First story detection structures are created on the basis of documents as vectors within a duration using term frequencies [31, 32. Distance measurement is used to detect first story, this is obtained by comparing new documents to their nearest neighbour by measuring their distance gap. Documents with distance that exceed a predefined maximum value are considered as first story. This method collects all document term frequencies in memory and detects the nearest neighbour for 
in-coming documents [33]. Tweets pertaining to a planned real life event are distinguished from the stream of non-event tweets [19] using an incremental online clustering algorithm. This scalable algorithm clusters a huge volume of Twitter messages without prior knowledge of the number of clusters. An incremental clustering algorithm is applied during training phase to place each message in a related existing cluster. Any new message that is not similar to the ones in an existing cluster forms a new cluster [19. An improved Locality Sensitive Hashing (LSH) was proposed by Petrovic et al [34] to search for nearest neighbour enhancement that satisfies the data stream mining prerequisites using constant size buckets. Osborne et al, 23. presented a method that merges Twitter and Wikipedia in order to enhance event detection. They explore the latency between the two streams and discovered that Twitter is more up-to-date in real life events posting. The authors of Weiler et al 35] presented a run-time and task-based performance of diverse event detection techniques for Twitter by correlating both, the run-time and task-based performance. For run-time performance they based their approach on a general-purpose data stream management structure. On the other hand, task-based performance was automatically determined having considered a string of novel measures. Their study looked into the implementations of advanced Twitter event detection techniques that 135 are relatively logical. Also, they gave a platform-based approach that points to more methodical performance studies for novel techniques for event detection in the future. Similarly in Weiler et al [36 they defined evaluation measures to significantly assess the precision and recall of event detection techniques for Twitter network. They derived a query plan using various state-of-the-art event detection techniques and demonstrated the ability of their proposed measures in evaluating the different techniques using real life Twitter data. The work of Guille \& Favre [37] proposed MABED (Mention-Anomaly-Based Event Detection), a statistical technique that depends completely on tweets and takes advantage on the creation frequency of dynamic links such as mentions often included in tweets to ascertain important events and to measure the degree of their impact to the crowd. MABED is said to dynamically estimate the time 
each event is discussed to also interprets the detected events by way of offering distinctive textual description and exact temporal descriptions. On the other hand, the work of McMinn \& Jose [38] applied a clustering method that partitions documents according to the groups they encompass, and 'burst' detection and cluster selection methods to discover clusters similar to an on-going real life event. Even though their technique was able to detect real life events while boosting precision and lowering computational complexity, their approach was not effective with tweets' hastags. Since hashtag is an integral part of online tweets, our approach considered the importance of hashtag very keenly by developing a system that automatically detects and tracks real life topics and events through hashtag labels of online tweets. Corney et al 39] used n-grams and term frequency-inverse document frequency $\left(d f-i d f_{t}\right)$ to group together terms that appear in the same tweets with hierarchical clustering. They identified term clusters whose similarities are high as a representation of same topic and merged clusters to the point where each cluster is assumed to signify a distinct topic. They presented more detail of their algorithm in Aiello et al [1] by identifying real world topics in the 2012 US Presidential Elections, the US Super Tuesday 2012 and the English FA Cup 2012. Different compared with approaches reviewed in this paper, our method is able to detect real life events through hashtags used in tweets relating to the specified events by identifying $A R$ present in tweets hashtags. Where other methods differentiate event tweets from non-event tweets by clustering [30, 28, our method detects and lists related hashtags of landmark events as they unfold. Our system also detects changes in $A R s$ patterns in hashtags at time $t$ and $t+1$ which demonstrates the evolvement of related real life topic/event. A weakness of our methods lies in the fact that it does not detect and track topic/event in real time, which we intend to consider in our future work. However, our evaluation reveals how the dynamics of events from different domain affects our experimental results. Sports events (especially football) is a short-term and relatively emergent events, while political events are long-term and stable events. The experiments in this paper highlights the relevance of tweets' hashtags as a pointer 
to tweets' content.

$\begin{array}{ll}n & \text { Table 1: Notation of Terms } \\ r_{i}^{t} & \text { number of hashtags } \\ & \text { a set of all rules generated at time } t \text { where } i \in\{1, \ldots, \mid \\ r_{j}^{t+1} & \text { a set of all rules generated at time } t+1 \text { where } j \in\{1, \ldots, \\ l h_{i} / l h_{j} & \left.r^{t+1} \mid\right\} \\ & \text { number of hashtags with value } 1 \text { present in conditional } \\ r h_{i} / r h_{j} & \text { nurt of rule } i \text { and } j \\ & \text { part of rule } i \text { and } j \\ l h_{i j} / r h_{i j} & \text { number of matching hashtags in conditional/consequent } \\ & \text { part of rules } i \text { and } j \\ p_{i j} / q_{i j} & \text { degree of similarity of hashtags in conditional/conse- } \\ & \text { quent part of rules } i \text { and } j \\ t h p_{i j} / t h q_{i j} & \text { Threshold of degree of similarity of hashtags in condi- } \\ & \text { tional/consequent part of rules at } t \text { and } t+1\end{array}$

\section{Notation of Terms}

In Liu et al [40] and Song et al 41] similar methods for calculating similarities and differences between two rules in relational datasets to detect association rules at two different times were employed. The method used in this paper defines the similarity based on the principles of degree of similarity proposed in previous work [40, 41]. Details of the calculations and notation used are stated in Table. 1 .

\subsection{Identifying Association Rules in Tweet Hashtags}

$A R M$ is a data mining technique used for mining significant ARs common to different collections of items in data warehouses such as transactional and relational databases [42, 40]. ARM evaluates the frequent antecedent/consequent 

relationships [43] that satisfy the user-defined support/confidence threshold. Support shows the frequency of the items in the database while confidence shows the number of times the frequency likelihood is positive. For example, the likelihood that a buyer who purchases bread stands $80 \%$ chance of purchasing

patterns by using the support and confidence measures to detect significant butter. The downward closure property of frequent patterns implies that any subset of a frequent itemset must be frequent. This criterion is necessary for pruning the search space during each iteration of the Apriori algorithm. In this work, we choose a low minimum support to exclude the problem of missing not so frequent but important items in the datasets. The Apriori method is a common algorithm for learning ARs [4]. It detects frequent itemsets and strong ARs 45]. The application of Apriori to tweet hashtags at two time periods $t$ and at $t+1$ as presented in Fig. 1 produces two association rulesets which we interpret as rules evolvement in the context of this work. In our previous paper [12], TRCM was used to identify four (temporal) dynamic rules in tweets hashtags namely; "New rules" (N), "Unexpected Consequent" rules (UnxCs)/ "Unexpected Conditional" Rules (UnxCn), "Emerging" rules (EM) and "Dead" rules (D). The rules were acquired by matching rules present in tweets at the two periods under study, $t$ and $t+1$. Rule Matching Threshold (RMT) for degree of similarity in the conditional part of rules $\left(p_{i j}\right)$ and in consequent part of rules $\left(q_{i j}\right)$ are assigned between 0 and 1 , with 1 indicating maximum rule similarity and 0 indicating maximum rule dissimilarity as presented in equ. 1 .

$$
\begin{gathered}
R M T=t h p_{i j}=t h q_{i j} \\
p_{i j} \in[0,1], q_{i j} \in[0,1] \\
\text { Where }: i \in\left\{1,2,3, \ldots\left|r^{t}\right|\right\} \\
j \in\left\{1,2,3, \ldots\left|r^{t+1}\right|\right\}
\end{gathered}
$$

The degree of similarity/dissimilarity measure is built to detect the degree of change in rules. The changes are then grouped under the four identified rules. 




Figure 1: TRCM process

215 TRCM revealed the dynamics of $A R$ present in tweets and demonstrates the linkage between the different types of rule dynamics investigated in the form of rule evolvement/trend as will be discussed in Section 5

3.2. Rule Matching Equations

$$
\begin{aligned}
p_{i j} & =\frac{l h_{i j}}{\max \left(l h_{i}, l h_{j}\right)} \\
q_{i j} & =\frac{r h_{i j}}{\max \left(r h_{i}, r h_{j}\right)}
\end{aligned}
$$

Equations 2 and 3 demonstrate the similarity in both, the conditional $p_{i j}$ and consequent $q_{i j}$ parts of rule $i$ and rule $j$ at time $t$ and $t+1$ respectively as 


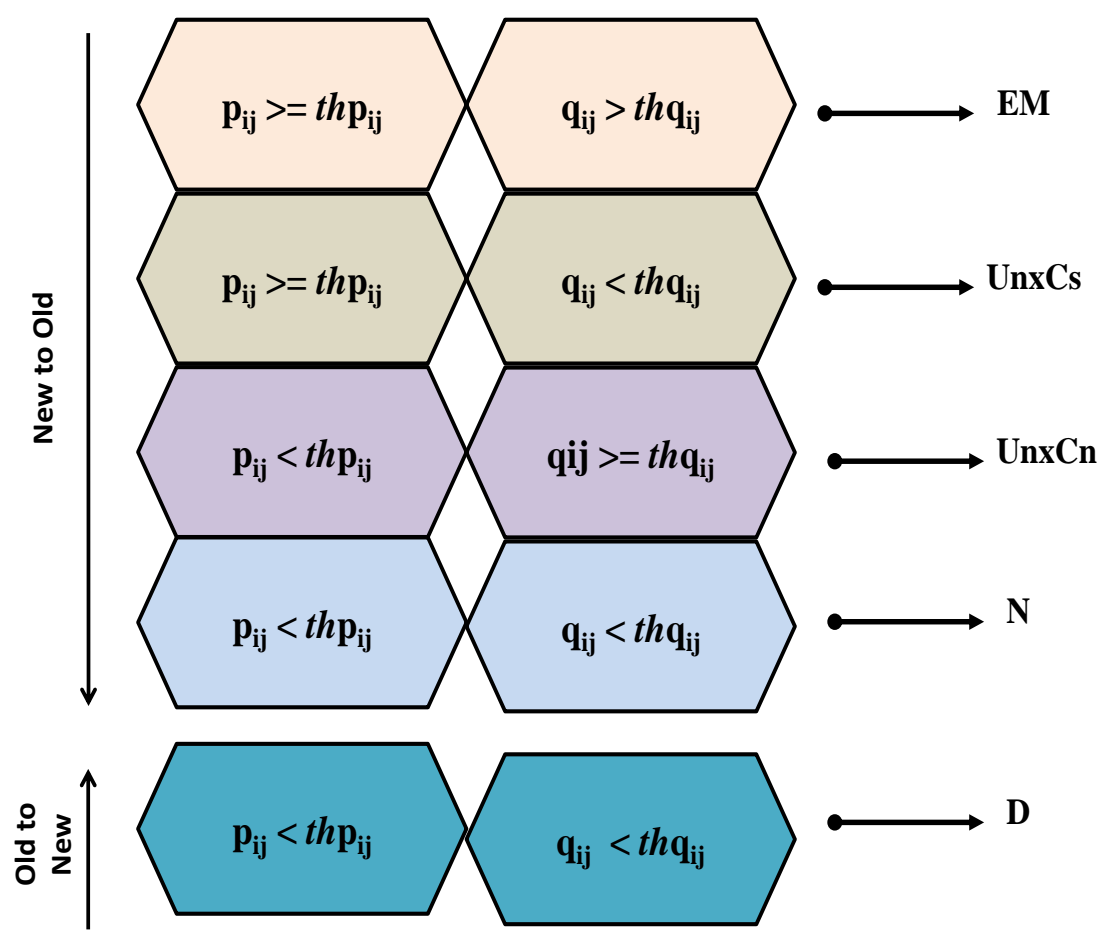

Figure 2: TRCM rules assignment

shown in Fig. 2. The equations are adopted from Liu et al [12]. Change in rules can be discovered by matching every rule in ruleset $r_{i}^{t}$ with those in ruleset $r_{j}^{t+1}$ using the similarity and difference comparison.

However, for two rules to be similar, their degree of similarity must be greater 225 than the pre-defined Rule Matching Threshold (RMT) whose maximum value is 1 .

Similarity Measure $=$ Degree of similarity between $r_{i}^{t}$ and $r_{j}^{t+1}$;

$$
\left(0 \leq p_{i j} \leq 1,0 \leq q_{i j} \leq 1\right)
$$

Where degree of similarity is less than the RMT, the rules are said to be 230 different. 


\section{Development of TRCM Architectural Framework}

TRCM framework was built using the Apriori method of ARM. The framework defines patterns of $A R s$ changes in tweets at different periods in relation to similar real life scenario. To build TRCM framework, the left hand side $t h$ -

$235 \mathrm{~s} /$ conditional and the right hand side rhs/consequent parts of rules in Apriori principle were employed to analyse hashtags present in tweets. The evaluation of the lhs and the rhs is used to identify the $A R s$ present in tweets at different times. The similarities and differences in the $A R$ in the rulesets $r_{i}^{t}$ and $r_{j}^{t+1}$, (where $t$ is the time and $i, j$ are rules present in tweets at $t$ and $t+1$ respectively) are measured to determine TRCM rules namely; "Emerging", "Unexpected", "New" and "Dead" rules in tweets.

\subsection{Definitions of TRCM Rules}

Rule Matching in rulesets at $t$ and $t+1$ results in the definition of TRCM rule change patterns. An Unexpected Consequent rule arises when a rule in $r_{i}^{t}$ and $r_{j}^{t+1}$ has a similar conditional part but different consequent part $\left(p_{i j} \geq t h p_{i j}\right.$ and $\left.q_{i j}<t h q_{i j}\right)$ compared with an existing rule.

- \#flightMH370 $\Rightarrow$ \#missing (Ruleset at time $t$ )

- \#flightMH370 $\Rightarrow$ \#TimAkers (Ruleset at time $t+1$ )

An Unexpected Conditional rule is detected when the consequent parts of rule $r_{i}^{t}$ at and $r_{j}^{t+1}$ are similar, but the conditional parts are different $\left(p_{i j}<\right.$ $t h p_{i j}$ and $q_{i j} \geq t h q_{i j}$ ). Having described unexpected consequent rule change in real life situation, it is important to mention that both unexpected consequent and unexpected conditional rule change in real life are presented in the same way. An example of an unexpected rule in real life is sudden event occurrence. For example in the case of the missing Malaysia flight, a claim by the British marine archaeologist Tim Akers of having found MH370 3,000 miles from the search zone after spotting debris painted in the colours of Malaysia Airlines can result in unexpected rule change. 
- \#Malaysia $\Rightarrow \#$ flightMH370 (Ruleset at time $t$ )

- \#Missing $\Rightarrow \# f l i g h t M H 370$ (Ruleset at time $t+1$ )

Emerging rules occur when rules at time $t$ and $t+1$ have similar conditional and consequent parts of the rule with similarity greater than the user-defined threshold $\left(p_{i j} \geq t h p_{i j}\right.$ and $\left.q_{i j}>t h q_{i j}\right)$. Instances of a real life events that may generate an emerging rule in $T R C M$ are for example global breaking news of a disaster or the announcement of the US presidential elections winner.

- \#Missing $\Rightarrow$ \#flightMH370 (Ruleset at time $t$ )

- \#Missing $\Rightarrow \#$ flightMH370 (Ruleset at time $t+1$ )

Breaking news often generate high volume of tweets very rapidly resulting not classified as one of the three previous types of rules (emerging, unexpected consequent and unexpected conditional rules) are classified as new rules. A rule in $t$ is classified "dead" if its maximum similarity measure with all the rules in $t+1$ is less than the user-defined threshold. "Dead" rule in real life are topics ${ }_{275}$ that were initially tweeted but were no longer visible in Twitter network after some time.

In this work, we apply different $T R C M$ rules to each of the three datasets used in our experiments. First, we analyse the datasets by applying only the unexpected rules (consequent and conditional). Next, we apply only the emerging rules and finally, we analyse the datasets using the combination of both the unexpected and the emerging rules. We evaluate the performance profile of each of the TRCM rules when applied autonomously on the datasets and the degree of performance enhancement when both rules are combined.

\section{Trend Analysis of Rules in Tweets Hashtags}

285

Experimental investigations conducted in [12, 13] show that ARs present in tweets' hashtags evolve over time. This results in what is referred to as 
rule trend. Trend Analysis (TA) in the context of our work, is a way of analysing the trend (evolvements) of TRCM rules identified in tweets and displayed by hashtag keywords over a specified period of time. The concept of $T A$ (days or months) depending on the domain to which the topic belongs. A rule $\#$ Drogba $=>$ \#goal in a football event may evolve into \#Drogba $=>$ \#yellowcard within the following minute. This evolvement implies that Drogba scored a goal and in the next minute, he was booked for foul play. In politics, a rule \#Obama $315 \Rightarrow$ \#Ohio may take 5 hours to evolve into \#Obama =>\#victoryspeech. In this case, the first rule may have been detected when Obama won the poll in Ohio 
and the second rule detection when he gave his victory speech five hours later. A rule may start up as an emerging rule based on the dynamics of the topic involved (for example breaking news), another rule may display only the "New" status and become "Dead" shortly afterwards. Sequence A in Fig. 3 demonstrates reverse trend in rule (the rule evolved back into an emerging state) before becoming a "Dead" rule. Sequence $B$ reveals that the rule came into the network as an unexpected consequent rule and maintains the status all through its lifespan on Twitter. Lastly sequence C presents an "Emerging" rule that maintains its emerging status without becoming a "Dead" rule. In real life, such a rule can be related to consistent news topics such as foreign exchange in business news. Sequences of TFWs demonstrate the relevance of real life events. This can be justified by the pattern of their ARs sequences of evolvements and how long specific evolvement statuses are retained. We present the notation for sequence of evolving rules in Table. 2

Table 2: Sequences of Evolving Rules

$T \quad$ The total time period intervals a rule status is measured in.

$C_{t} \quad$ The category of the rule

$C_{t} N \quad$ New rule

$C_{t} U_{t}^{i} \quad$ Unexpected conditional rule

$C_{t}^{j} t \quad$ Unexpected consequent rule

$C_{t} E \quad$ Emerging rule

$C_{t} D \quad$ Dead rule

$T F W \quad$ Number of frame window

$T A$ of rules evolvements in tweets can be adapted by entities such as journalists and news media for effective news reports of news updates. Having explained how rules are introduced and how they evolve on Twitter, next we describe how a topic originates in relation to Twitter and the traditional newsagents in reality. 

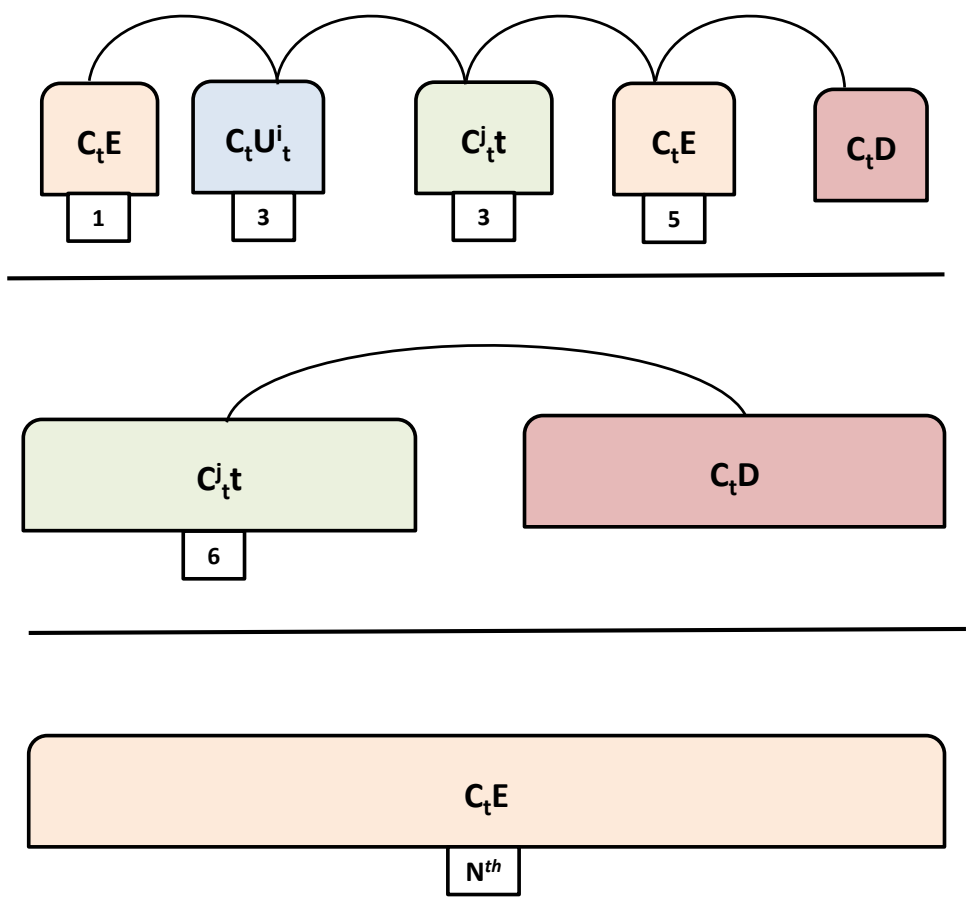

Figure 3: Time Frame Sequences of evolving rules

\subsection{The "TwO - NwO - EvO" State of Trend Analysis}

Twitter users are known to tweet about an event in real time. Such tweets may trigger the broadcast of the event (i.e. by newsagents) as illustrated in Fig. 4. For example, the news of the death of Whitney Houston was believed to have been tweeted some minutes before it was broadcasted by traditional newsagents.

In this work, we describe tweets that come before the broadcast of its related news in real life as $\boldsymbol{T} \boldsymbol{W}$ eet $\boldsymbol{O}$ riginated topic or $\boldsymbol{T} \boldsymbol{w} \boldsymbol{O}$ topic. On the other hand, event broadcasted by newsagents may result in keywords relating to the event being hashtagged in online tweets either in the form of opinion expression or information dissemination via the Twitter network. In this case the topic is 


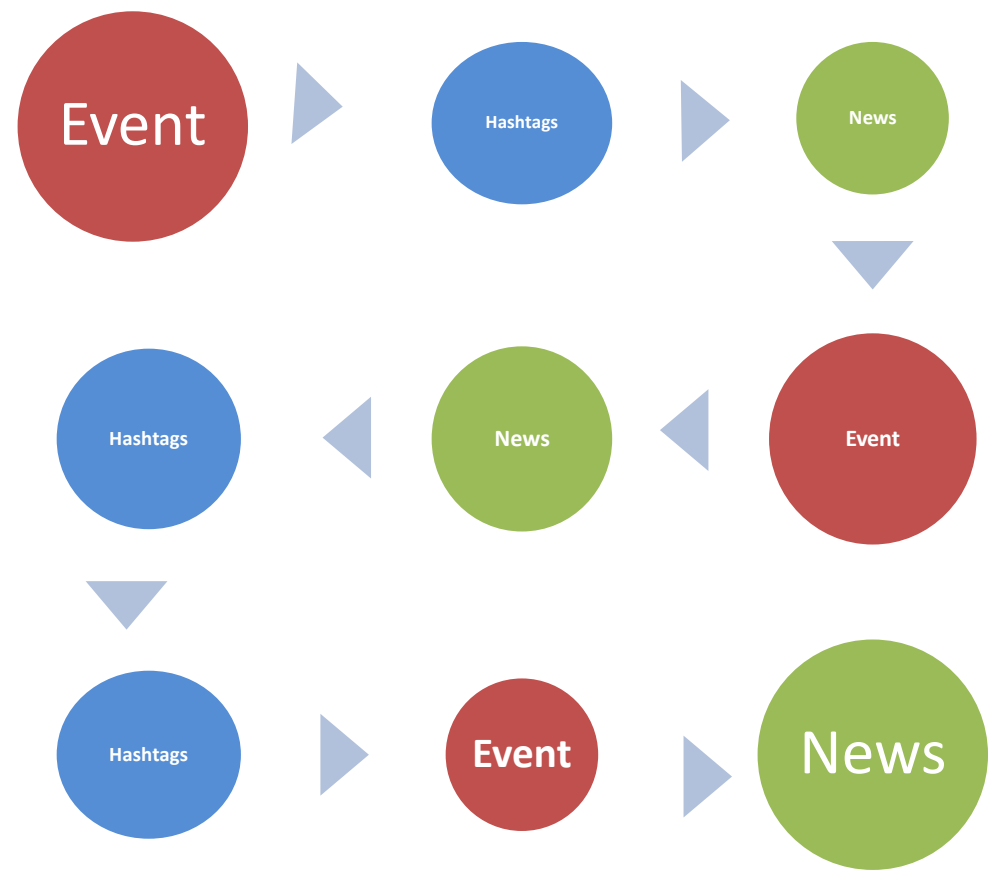

Figure 4: "TwO - NwO - EvO" states of Trend Analysis

said to be $\boldsymbol{N e W s} O$ riginated or $N w \boldsymbol{O}$ topic. Apart from these two states, a planned event can be hashtagged on Twitter for the purpose of publicity even before the event takes place. We refer to this type of situation as Event $O$ riginated topic or $\boldsymbol{E} \boldsymbol{v} \boldsymbol{O}$ topic. The three datasets used in our experiments fall under the $E v O$ topic.

\subsection{Detecting Real Life Topics from Tweets' Hashtags - Problem Definition}

The main focus of our experiments is to extract real life (newsworthy) topics from tweets hashtags of any domain using TRCM. Hashtags are principally meant to place emphasis on significant keywords in tweets or give title to online tweets. As mentioned earlier, the inclusion of hashtags in tweets enhances the chance of the readability of such tweets. This requires the development of a 
framework that will serve as a TDT tool for extracting evolving $A R s$ of hashtags from event tweets and mapping them to the ground truth within the relevant time window. The combined use of hashtags and ARM is a novel TDT method when compared to existing TDT methods used for analysing Twitter data. To detect newsworthy topics from tweets, users begin by supplying hashtag keyword(s) that best describe a specific topic to the Twitter streaming API in order to filter and collect only tweets that include the specified hashtag keywords. Recently it has become common for different entities, including event organisers and newsagents, to provide an official hashtag that describes tweets relating to their event, for example \#Supertuesday, \#Elections2012 and \#F ACup were official hashtags of the datasets used in our experiments. We determine the length of time to be considered for analysis and specify the update interval of 370 evolving ARs that were mapped to the chosen ground truth.

\section{Twitter Datasets: Methods for Collection and Preprocessing}

Our methodological process begins from the description of datasets used for the experiment as described next.

\subsection{Datasets}

The English Football League is a popular and important tournament in English football games. The event is viewed all over the globe with fans of English football clubs spanning around the world. The tournament marks the peak of several divisional leagues with the winners of each division advancing to participate in the FA Cup finals game. The 2012 FA Cup finals featured Chelsea Football Club and Liverpool Football Club, with both teams having huge amounts of fans in and outside the UK. Expectedly, fans of the two teams tweeted about the match before, during and after the match was played. Chelsea Football Club won by 2 goals to 1 .

The US Presidential Elections was conducted in November 2012 with Barack

Obama representing the Democratic Party and Mitt Romney representing the 
Republican Party. The election result reinstated the incumbent US president and his running mate for a second term in office.

In the United States Electoral System, Super Tuesday refers to the Tuesday in February or March of a presidential election year when the majority of the states conduct the primary elections. In these elections delegates are selected to national conventions where presidential candidates for each party are officially nominated. The Super Tuesday 2012 was held on March 6 in States such as; Alaska, Georgia, Idaho, Massachusetts, North Dakota, Ohio, Oklahoma, Tennessee, Vermont and Virginia, amongst others. The Super Tuesday tweets collection we used for the experiments considered keywords such as the four main Republican candidates namely, Mitt Romney, Ron Paul, Newt Gingrich, and Rick Santorum as well as the ten states and the major newsagents reporting the events.

TRCM system is trained to discover ARs present in tweets hashtags of selected datasets. We map hashtag keywords contained in the ARs obtained to related real life topics provided by Aiello et al [1].

\subsection{Data Collection and Preprocessing}

We use a collection of tweets relating to the 3 topics (FA cup final 2012, US elections 2012 and Super Tuesday 2012). These collections include main keywords particularly related to each topic. We extract the tweets and the hashtags with their timestamps as shown in Fig. 5. The timestamps enable us to map the time slot of hashtag keywords detected by TRCM to the event's time period in the ground truth. The FA Cup collection has about 444,291 tweets over a period of 72 hours (4 - 6 May 2012), however, we analysed only $50.6 \%(224,291)$ of the total collection. This represents the number of tweets posted online during the game (May 5 2012, 5:15pm to 7:00pm). For the US Election 2012 and the Super Tuesday, there were collections of about 3,837,291 and 474,109 respectively. 
slots with a 1 minute update rate due to the rapid evolvement rate of the game (where 2 goals can be scored within 2 minutes or less). For the US Presidential Elections 2012, we divide the tweets into roughly 20000 tweets/time slots with a 10 minute update rate. For the Super Tuesday we divided the tweets into roughly 10000 tweets/time slot and 1 hour update rate, because it was observed that events in political datasets evolve less frequently.

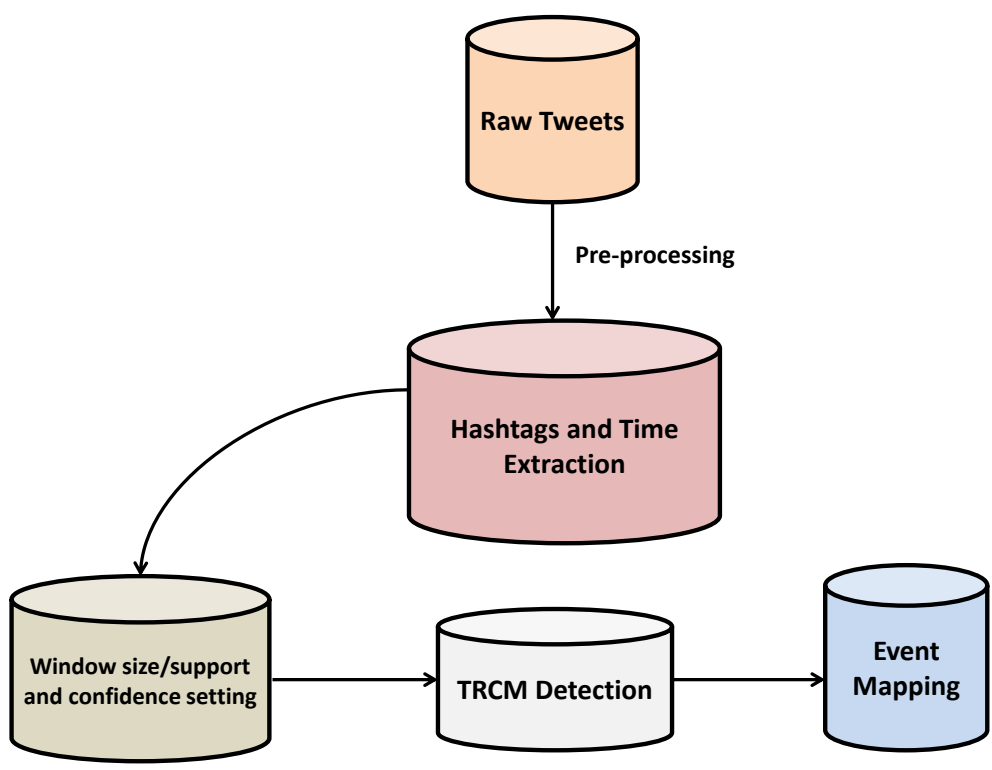

Figure 5: Event Detection Process

\section{Experimental Set-up}

Since hashtags usage on Twitter network is to describe tweets' content, we set out to conduct TDT experiments that automatically detect real life topics from to smaller chunks according to a pre defined window size and specify their update rate. For the English FA Cup finals we select 1 minute update period, for the US Presidential elections and Super Tuesday we select 10 minutes and 1 hour respectively as in Aiello et al [11. These settings were found to yield better 430 results on the datasets after empirical fine tuning. This setting enhances the precision of rules returned by $T R C M$ within each time-slot. We also set both, 
the support and confidence to 0.001 after carrying out a preliminary study to confirm that this is the setting that best optimises TRCM's efficiency on the case study datasets. In these experiments, we are more concerned with extracting as many relevant hashtag keywords relating to targeted real life topics/events (precision) as possible.

We extract all hashtags in the tweets and defined a function that finds equal terms in $l h s$ and rhs (left-hand side and right-hand side) rules of $r_{i}^{t}$ and at $r_{j}^{t+1}$. These are used to set the TRCM rules. We find matching values in $l h s$ ${ }_{440}$ and $r h s$ of $r_{j}^{t+1}$ and $r_{i}^{t}$ as presented in Fig. 6 where \#tcot and \#RonPaul are unexpected consequent rules (similar lhs but different rhs) and \#Romney is a new rule (no matching found). TRCM rules is detected by defining the $t h p_{i j}$ and $t h q_{i j}$ (left hand side and right hand side user-defined threshold), which are set between 0 and 1. The experiments were conducted in R Studio Version 3.0.0 (2013-04-03), Platform: $x 86 \_64-w 64-$ mingw32/x64 (64-bit) and processed on Windows 7 Enterprise of 8.00 RAM memory size and CPU @ 3.20GHz.

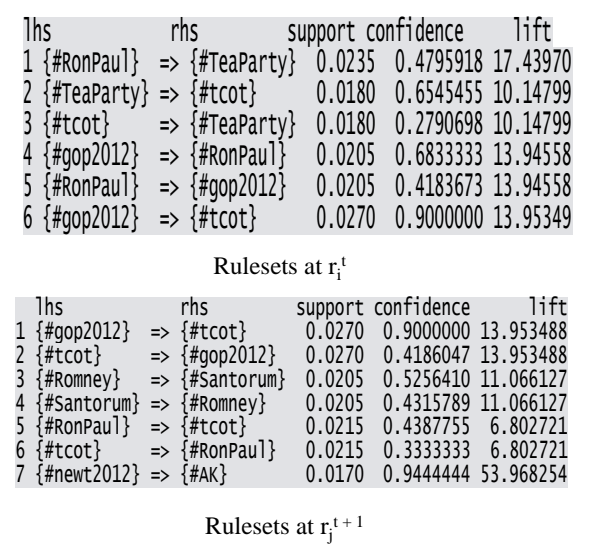

Figure 6: Rules Matching Sample

Events mapped by our system in the sports dataset include goals, bookings, substitutions, shot-on-targets, free kicks and foul plays. In the US Elections 2012 , events detected include the disruption of the two candidates' election campaign by Hurricane Sandy, California death penalty ban rejected by voters, the presidential election result and Obama's victory speech. In the Super 
Tuesday, events detection includes the election results in different states of the United States. For the experiments, an item $h$ is any hashtag present in the tweet, while the transaction is the tweet message that occurs in a time slot $T$. The number of times that any given set of hashtags occurs in the time slot is referred to as its support, and itemset (hashtag) that meets a minimum support is referred to as a pattern. To confirm event detection in the datasets, we examine hashtag keywords present in the ARs returned within each time-slot and rank them at 3 levels. First, we analyse hashtag keywords under the unexpected consequent/ unexpected conditional rules, then those under the emerging rules. Finally, we combine both, the unexpected and the emerging rules. All the hashtags detected in each time-slot at the 3 levels were recorded along with the time the tweets were posted on Twitter to evaluate our system's performance. We establish a match if the returned hashtags in each time-slot contain at least one of the key terms used in the ground truth within the same time frame the detection occurred as shown in Tables 4 and 5 We confirmed that the hashtag keywords detected as unexpected and emerging rules were those that best represent different event highlights in the datasets when mapped to the ground truth.

\section{Experimental Result}

To validate our topic detection technique we generate ground truth from Main Stream Media (MSM) for the 2 political datasets and for the sports dataset we generate ground truth from the BBC sports official website (http://goo.gl/Ir3Of). The FA Cup final match between Chelsea Football Club and Liverpool Football Club produce event highlights that were detected by TRCM. Our system was able to detect events such as goals scored, bookings, player substitutions, free kicks, offside, misses, saves and clearances. Event detection mapping was carried out manually as presented in Table. 3. For the US Elections 2012 our system mapped 11 out of 24 topics to the ground truth (45\%). We show samples of our system detection in Table. 3. The effectiveness measure for our 
system is discussed in the next section.

Table 3: Table Showing TRCM Event Detection for FA Cup 2012 Dataset

\begin{tabular}{|l|l|l|l|l|l|l|l|l|l|l|}
\hline & G & Sub & BK & FK & S & CL & OS & BL & MS & TE \\
Ground Truth & 3 & 4 & 3 & 10 & 11 & 19 & 4 & 3 & 2 & 59 \\
TRCM & 3 & 3 & 1 & 10 & 9 & 19 & 4 & 3 & 2 & 54 \\
\hline
\end{tabular}

In Table.1 GT $=$ Ground Truth; $\mathrm{G}=$ Goals; Sub = Substitutions; BK $=$ Bookings; FK $=$ Free kicks; $\mathrm{CL}=$ Clearances; OS = Offsides; $\mathrm{BL}=$ Blocks; MS $=$ Misses; TE $=$ Total Event

\subsection{Performance Measures}

Recall and precision are performance measure metrics used in Information Retrieval (IR) to evaluate the performance of an IR system. Precision is the percentage of relevant instances identified by the system, while recall is the percentage of relevant instance classified correctly [46, 47. The system error rates are used to evaluate appropriateness of the system. Other single-valued measures have been implemented [15]; however, F-Measure, which is the weighted harmonic mean of precision and recall is the most dominant approach for evaluating text classification.

In the experiments highlighted in this section, we measured the performance of our system by applying precision, recall and F-Measure to the three datasets. In summary, we classified all identified hashtag keywords as unexpected and emerging rules in each of the datasets and mapped them to the ground truth at three levels of Performance Variation (PV). First we mapped keywords of unexpected rules only, then we mapped emerging rules only and lastly, we mapped the combination of both unexpected and emerging rules as shown in Tables 67 and 8 . The purpose of this was to demonstrate the effectiveness of each mapping combination. The $P V$ shows that the application of both unexpected and emerging rules on the datasets enhanced the performance of our system particularly on the sports dataset. This can 


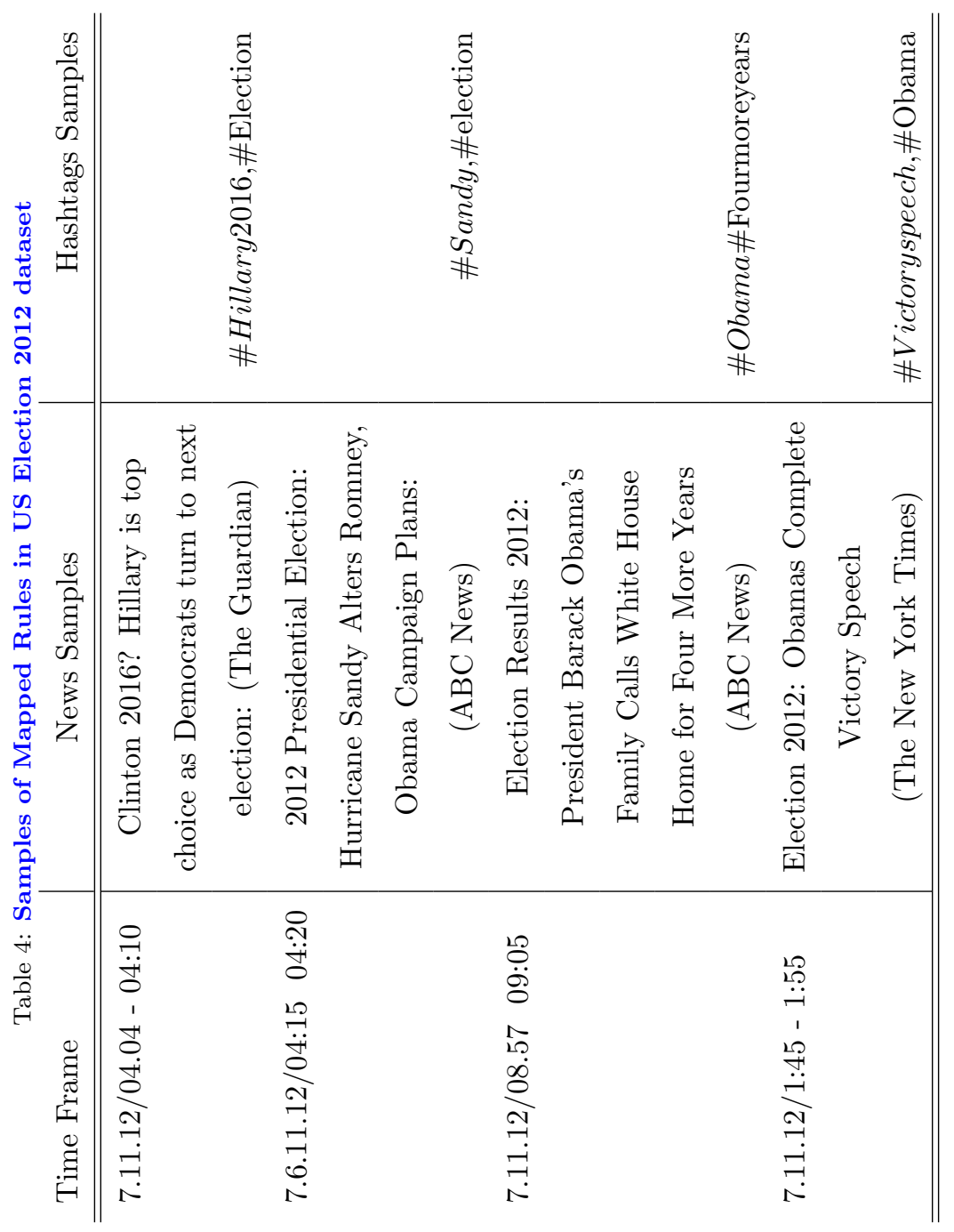




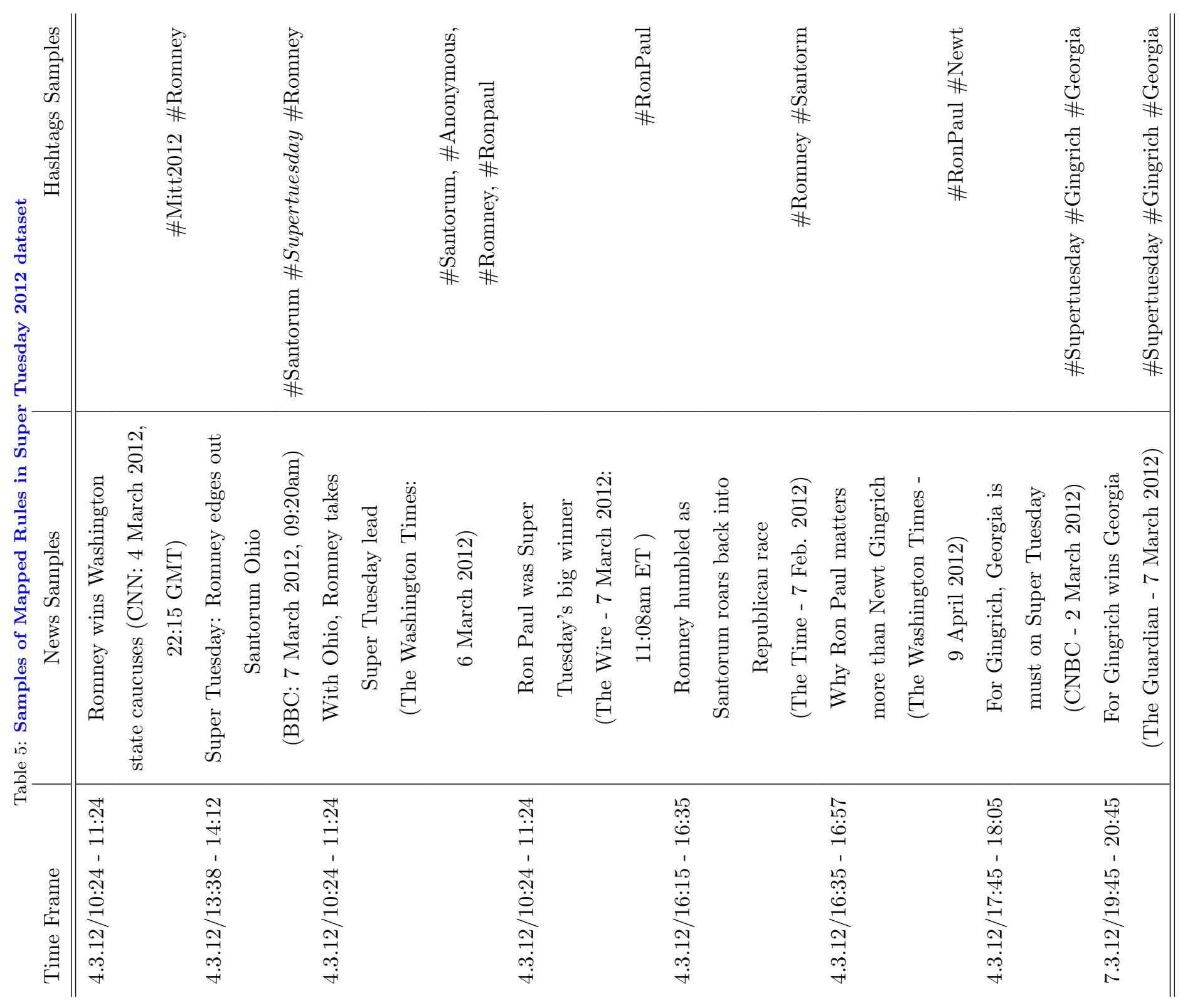


events (90 minutes to 120 minutes in the case of a football game). On the other hand, events in politics often have a longer timeline, thereby making event/topic detection and tracking more complex. Furthermore, the ground truth we used for the FA Cup 2012 presents one topic per time slot, whereas topics in the political datasets occurred in parallel. While the sports event ground truth covers all the highlights of the game, those for the two political datasets did not capture some of the important events that occurred during the US Elections 2012 and the Super Tuesday 2012.

Table 6: Table showing Precision PV

\begin{tabular}{cccc} 
Dataset & Unexpected & Emerging & Both \\
\hline \hline FA Cup & $91.5 \%$ & $5.8 \%$ & $96.6 \%$ \\
US Election & $34.6 \%$ & $19.2 \%$ & $53.8 \%$ \\
Super Tuesday & $37.5 \%$ & $25 \%$ & $62.5 \%$ \\
\hline \hline
\end{tabular}

Table 7: Table showing Recall PV

\begin{tabular}{cccc} 
Dataset & Unexpected & Emerging & Both \\
\hline \hline FA Cup & $85.7 \%$ & $3.89 \%$ & $64.0 \%$ \\
US Election & $40.9 \%$ & 20.8 & $70.0 \%$ \\
Super Tuesday & $20 \%$ & $14.2 \%$ & $55.5 \%$ \\
\hline \hline
\end{tabular}

Table 8: Table showing F-Measure PV

\begin{tabular}{cccc} 
Dataset & Unexpected & Emerging & Both \\
\hline \hline FA Cup & $88.5 \%$ & $4.40 \%$ & $76.9 \%$ \\
US Election & $37.4 \%$ & $19.96 \%$ & $60.0 \%$ \\
Super Tuesday & $26 \%$ & $18.1 \%$ & $58.79 \%$ \\
\hline \hline
\end{tabular}




\section{Discussion and Conclusion} formed better on dataset in the sports domain. This can be attributed to the short timeline for sporting events (90 minutes to 120 minutes in the case of a football game). On the other hand, events/topics in politics are known to have longer timeline making event/topic detection and tracking more complex. The performance measures used, namely precision, recall and F-Measure by up to $30 \%$ for the political datasets.

Twitter has become an important network to different entities in many ways. To individuals it has become a tool for raising awareness on diverse issues. It 540 ground truth we used for the FA Cup 2012 presents one topic per time slot whereas topics in the political datasets occurred in parallel. While the sports event ground truth covers all the highlights of the game, those for the two political datasets did not capture some of the important events that occurred during the US Elections 2012 and the Super Tuesday 2012. Further investigations into the hashtags keywords classified as false positives according to the ground truth, are found as news headlines on the websites of other newsagents. An example of such headlines is the one reported on the CNN websites under the title, "California Proposition 34: Ban Death Penalty" 1 and Huffington Post of November 7, 2012 under the title, "California Death Penalty Ban Rejected By Voters" 22. We also consider Wikipedia databases for US Elections and Super Tuesday 2012, during the US elections 2012, Wikipedia collected and posted online, all the elections results illustrated in texts, tables and graphs, making it easy to retrieve vital and credible information about the events. With these findings, we established that if we consider multi ground truth for our analysis, TRCM will exhibit enhanced results across all the three has also become a medium of information dissemination, which includes breaking news. Individuals visit the network to search for opinions of other people

\footnotetext{
${ }^{1}$ http://edition.cnn.com/election/2012/results/state/CA/ballot/02/

${ }^{2}$ http://www.huffingtonpost.com/2012/11/07/california-death-penalty-_n_2090260.html
} 
on products, services or even national issues. Similarly, business organisations keep an eye on activities on Twitter to know how their stakeholders perceive their business. Most government bodies now relate with their subjects through Twitter and encourage them to post their opinions on the network. The enormous data generated on the network requires data mining techniques such as ARM to analyse and classify tweets, in order to detect their similarities and differences in relation to event patterns. The rule dynamics of ARM shows that tweets go through different phases. We proposed the Transaction-based Rule Change Mining (TRCM) that detects rules changes based on hashtags present in tweets and how the changes relate to events/occurrences in real life scenarios. All the rules detected can be applied to the real world as a decision support tool for different entities, including individuals, organisations and government. potential crisis, such as increasingly bad reputation by monitoring all the conversations around their brands/organisations. Additionally, users could set up alerts in a second phase to be informed about these events in real-time and react before they have a big impact. As a result of such an alert, organisations can be aware of users/other organisations/media press steering the public opinion. This awareness can be derived through provisioning of insights about topics covered in those conversations and detection of sentiment polarity of the arguments in such conversations.

As we are dealing with open data sources, benchmark exercises are useful to compare and analyse the main events around a brand/company and competitors and search for similarities/patters and differences among the events in terms of users moving conversations, discussed topics, etc.

Future directions of the research reported in this paper can be identified as follows.

- With the "Moments" service launched by Twitter later in 2015, TRCM could be adopted to break down unfolding events as they happen, which 
would be of great interest to Twitter users.

- Applying TRCM to other social media networks like Facebook and LinkedIn could enable users to capture the dynamics of major events of interest in their personal and professional environment.

- Varying the granularity of the method in identifying development of events via emerging and unexpected rules by setting lower and/or higher threshold values for association rule interestingness measures (support and confidence) could enable zooming in and out through unfolding events. This could be used to personalise the outcome to the user's context (e.g., mobile users can rely on higher granularity of results, summarising events of interest, while desktop users with more time to examine the details could look at finer granularity of results.

- Adopting hashtag keywords found in emerging and unexpected rules as features for high performance classifiers such as Random Forests, Gradient Boosting trees, and/or Support Vector Machines could potentially identify/highlight individual tweets of interest to the user (i.e., breaking news).

\section{Bibliography}

[1] J. Li, A. Vishwanath, H. R. Rao, Retweeting the fukushima nuclear radiation disaster, Communications of the ACM 57 (1) (2014) 78-85.

[2] J. H. Lau, N. Collier, T. Baldwin, On-line trend analysis with topic models: \\# twitter trends detection topic model online., in: COLING, 2012, pp. $1519-1534$.

[3] M. Beaupierre, Social media stages of change-why moving to ?action? is right for public health, in: 142nd APHA Annual Meeting and Exposition (November 15-November 19, 2014), APHA, 2014. 
[4] D. L. Lasorsa, S. C. Lewis, A. E. Holton, Normalizing twitter: Journalism practice in an emerging communication space, Journalism Studies 13 (1) (2012) 19-36.

[5] N. Newman, The rise of social media and its impact on mainstream journalism, Reuters Institute for the Study of Journalism.

[6] C. Castillo, M. Mendoza, B. Poblete, Information credibility on twitter, in: Proceedings of the 20th international conference on World wide web, ACM, 2011, pp. 675-684.

[7] M. Cataldi, L. Di Caro, C. Schifanella, Emerging topic detection on twitter based on temporal and social terms evaluation, in: Proceedings of the Tenth International Workshop on Multimedia Data Mining, ACM, 2010, p. 4.

[8] S. Whiting, K. Zhou, J. Jose, O. Alonso, T. Leelanupab, Crowdtiles: presenting crowd-based information for event-driven information needs, in: Proceedings of the 21st ACM international conference on Information and knowledge management, ACM, 2012, pp. 2698-2700.

[9] M. Naaman, J. Boase, C.-H. Lai, Is it really about me?: message content in social awareness streams, in: Proceedings of the 2010 ACM conference on Computer supported cooperative work, ACM, 2010, pp. 189-192.

[10] J. Allan, Introduction to topic detection and tracking, in: Topic detection and tracking, Springer, 2002, pp. 1-16.

[11] L. M. Aiello, G. Petkos, C. Martin, D. Corney, S. Papadopoulos, R. Skraba, A. Goker, I. Kompatsiaris, A. Jaimes, Sensing trending topics in twitter.

[12] M. Adedoyin-Olowe, M. M. Gaber, F. Stahl, Trcm: a methodology for temporal analysis of evolving concepts in twitter, in: Artificial Intelligence and Soft Computing, Springer, 2013, pp. 135-145.

[13] J. B. Gomes, M. Adedoyin-Olowe, M. M. Gaber, F. Stahl, Rule type identification using trcm for trend analysis in twitter, in: Research and Development in Intelligent Systems XXX, Springer, 2013, pp. 273-278. 
[14] J. Guzman, B. Poblete, On-line relevant anomaly detection in the twitter stream: an efficient bursty keyword detection model, in: Proceedings of the ACM SIGKDD Workshop on Outlier Detection and Description, ACM, 2013, pp. 31-39.

[15] G. van Oorschot, M. van Erp, C. Dijkshoorn, Automatic extraction of soccer game events from twitter, in: Proc. of the Workshop on Detection, Representation, and Exploitation of Events in the Semantic Web, 2012.

[16] J. Ausserhofer, A. Maireder, National politics on twitter: structures and topics of a networked public sphere, Information, Communication \& Society 16 (3) (2013) 291-314.

[17] R. McCreadie, C. Macdonald, I. Ounis, M. Osborne, S. Petrovic, Scalable distributed event detection for twitter, in: Big Data, 2013 IEEE International Conference on, IEEE, 2013, pp. 543-549.

[18] P. Agarwal, R. Vaithiyanathan, S. Sharma, G. Shroff, Catching the longtail: Extracting local news events from twitter., in: ICWSM, 2012.

[19] H. Becker, M. Naaman, L. Gravano, Beyond trending topics: Real-world event identification on twitter., ICWSM 11 (2011) 438-441.

[20] M. Mathioudakis, N. Koudas, Twittermonitor: trend detection over the twitter stream, in: Proceedings of the 2010 ACM SIGMOD International Conference on Management of data, ACM, 2010, pp. 1155-1158.

[21] M. Naaman, H. Becker, L. Gravano, Hip and trendy: Characterizing emerging trends on twitter, Journal of the American Society for Information Science and Technology 62 (5) (2011) 902-918.

[22] S. Phuvipadawat, T. Murata, Breaking news detection and tracking in twitter, in: Web Intelligence and Intelligent Agent Technology (WI-IAT), 2010 IEEE/WIC/ACM International Conference on, Vol. 3, IEEE, 2010, pp. $120-123$. 
[23] M. Osborne, S. Petrovic, R. McCreadie, C. Macdonald, I. Ounis, Bieber no more: First story detection using twitter and wikipedia, in: Proceedings of the Workshop on Time-aware Information Access. TAIA, Vol. 12, 2012.

[24] A. Tumasjan, T. O. Sprenger, P. G. Sandner, I. M. Welpe, Predicting elections with twitter: What 140 characters reveal about political sentiment., ICWSM 10 (2010) 178-185.

[25] K. Watanabe, M. Ochi, M. Okabe, R. Onai, Jasmine: a real-time localevent detection system based on geolocation information propagated to microblogs, in: Proceedings of the 20th ACM international conference on Information and knowledge management, ACM, 2011, pp. 2541-2544.

[26] H. Becker, D. Iter, M. Naaman, L. Gravano, Identifying content for planned events across social media sites, in: Proceedings of the fifth ACM international conference on Web search and data mining, ACM, 2012, pp. 533-542.

[27] D. Inouye, J. K. Kalita, Comparing twitter summarization algorithms for multiple post summaries, in: Privacy, security, risk and trust (passat), 2011 ieee third international conference on and 2011 ieee third international conference on social computing (socialcom), IEEE, 2011, pp. 298-306.

[28] H. Sayyadi, M. Hurst, A. Maykov, Event detection and tracking in social streams., in: ICWSM, 2009.

[29] X. Meng, F. Wei, X. Liu, M. Zhou, S. Li, H. Wang, Entity-centric topicoriented opinion summarization in twitter, in: Proceedings of the 18th ACM SIGKDD international conference on Knowledge discovery and data mining, ACM, 2012, pp. 379-387.

[30] A. Jackoway, H. Samet, J. Sankaranarayanan, Identification of live news events using twitter, in: Proceedings of the 3rd ACM SIGSPATIAL International Workshop on Location-Based Social Networks, ACM, 2011, pp. $25-32$. 
[31] J. Allan, Topic detection and tracking: event-based information organization, Vol. 12, Springer, 2002.

[32] J. Yang, V. Honavar, Feature subset selection using a genetic algorithm, in: Feature extraction, construction and selection, Springer, 1998, pp. 117-136.

[33] P. Indyk, R. Motwani, Approximate nearest neighbors: towards removing

[38] A. J. McMinn, J. M. Jose, Real-time entity-based event detection for twitter, in: Experimental IR Meets Multilinguality, Multimodality, and Interaction, Springer, 2015, pp. 65-77.

[39] D. Corney, C. Martin, A. Göker, Spot the ball: Detecting sports events on the curse of dimensionality, in: Proceedings of the thirtieth annual ACM symposium on Theory of computing, ACM, 1998, pp. 604-613.

[34] S. Petrović, M. Osborne, V. Lavrenko, Streaming first story detection with application to twitter, in: Human Language Technologies: The 2010 Annual Conference of the North American Chapter of the Association for Computational Linguistics, Association for Computational Linguistics, 2010, pp. $181-189$.

[35] A. Weiler, M. Grossniklaus, M. H. Scholl, Run-time and task-based performance of event detection techniques for twitter, in: Advanced Information Systems Engineering, Springer, 2015, pp. 35-49.

[36] A. Weiler, M. Grossniklaus, M. H. Scholl, Evaluation measures for event detection techniques on twitter data streams, in: Data Science, Springer, 2015, pp. 108-119.

[37] A. Guille, C. Favre, Event detection, tracking, and visualization in twitter: a mention-anomaly-based approach, Social Network Analysis and Mining 5 (1) (2015) 1-18.

twitter, in: Advances in Information Retrieval, Springer, 2014, pp. 449-454. 
[40] D.-R. Liu, M.-J. Shih, C.-J. Liau, C.-H. Lai, Mining the change of event trends for decision support in environmental scanning, Expert Systems with Applications 36 (2) (2009) 972-984.

[41] H. S. Song, S. H. Kim, et al., Mining the change of customer behavior in an internet shopping mall, Expert Systems with Applications 21 (3) (2001) $157-168$.

[42] R. Agrawal, T. Imieliński, A. Swami, Mining association rules between sets of items in large databases, in: ACM SIGMOD Record, Vol. 22, ACM, 1993, pp. 207-216.

[43] A. Evfimievski, R. Srikant, R. Agrawal, J. Gehrke, Privacy preserving mining of association rules, Information Systems 29 (4) (2004) 343-364.

[44] A. Joshi, J. Sodhi, Target advertising via association rule mining, International Journal 2 (5).

[45] R. Srikant, Q. Vu, R. Agrawal, Mining association rules with item constraints., in: KDD, Vol. 97, 1997, pp. 67-73.

[46] R. Baeza-Yates, B. Ribeiro-Neto, et al., Modern information retrieval, Vol. 463, ACM press New York, 1999.

[47] R. ž Precision, Comments on ?data mining static code attributes to learn defect predictors?, IEEE Transactions on Software Engineering 33 (9) $725 \quad$ (2007) 635. 